\title{
STUDY ON THE EFFECT OF BROADBAND GAUSSIAN JAMMING TO FLL OF NAVIGATION RECEIVER
}

\author{
Wang Jun ${ }^{1, a}$, Hu Yu-Xing ${ }^{2, b}$ \\ ${ }^{1}$ Department of Electronic Engineering, Beijing Jiaotong University, Beijing 100044,China; \\ ${ }^{2}$ School of Information and Electronics, Beijing Institute of technology, Beijing 100081,China. \\ awangjun1@bjtu.edu.cn, ${ }^{\mathrm{b}} 13810433747 @ 163 . c o m$ \\ Supported by National Natural Science Foundation of China (61201199)
}

Keywords: FLL; broadband Gaussian noise jammer; navigation receiver

\begin{abstract}
Broadband Gaussian noise is a kind of important jammer to navigation receiver, for be regarded as the increase of the thermal noise of FLL usually, there is short of in-depth analysis to its effect to the performance of FLL. However, the effect of broadband Gaussian noise jammer to FLL is associated with frequency discriminator, filtering and other sectors. Based on considering the key sectors, the effect of broadband Gaussian noise jammer to the tracking error of FLL is studied in-depth, and the closed-form solution is derived. The simulation results show the correctness of the conclusion.
\end{abstract}

\section{Introduction}

For the power of navigation signal is very weak, the navigation receiver is sensitive to electromagnetic jammer, and then the tracking loop is easy to lose lock. The problem about the effect of jammer to the tracking loop of receiver has been attracting many researchers ${ }^{[1]}$. Through acquisition, the rough estimate of the carrier frequency can be obtained, but its error is still relatively large, lead to the low-order phase-locked loop(PLL) can not lock , then the error of frequency estimation is need to be drag into the linear working range of PLL by frequency-locked loop(FLL) generally ${ }^{[2]}$. Therefore, carrier recovery and tracking are the keys of the navigation receiver to work properly. The broadband Gaussian noise is a kind of important jammer to navigation receiver. To the effect of jammer on the navigation receiver performance, the reseachers pay more attention to the PLL ${ }^{[2-4]}$, but a little to the FLL, the FLL is often simply to be act as a special case of the PLL. The broadband Gaussian noise jammer is often viewed as additional thermal noise floor of receiver, and then the conclutions of the Ref. [5] is used to analysis the performance of FLL directly, then there is a short of in-depth analysis. Thermal Noise fibrillation is one of the main error sources to the FLL. The noise term of FLL is closely related to its frequency discriminator, then the error caused by noise fibrillation need to be anaysised according to the specific design of the loop. Based on considering the key parts of FLL, the effect of broadband Gaussian noise to the tracking error of FLL is studied in-depth, and the colsed form solution is derived. Based on the above analysis, the tracking threshold of FLL is got.

\section{The mode of FLL and signal}

For the higher dynamic and poor power of navigation signal, the classic structure of receiver is a second-order FLL assisted third-order PLL ${ }^{[5]}$. Then the object of study is second-order frequency-locked loop in this paper. The figure 1 shows the mode of signal and block diagram of the FLL. 


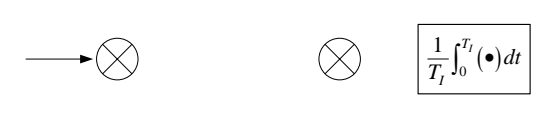

Fig.1 Block diagram of the FLL and the mode of signal

Where, $s\left(t_{n}\right)=\sqrt{2 P} D\left(t_{n}\right) P N\left(t_{n}-t_{s}\right) \cos \left(2 \pi f_{0} t_{n}+2 \pi f_{d} t_{n}+\varphi\right)$ is navigation signal, $P$ is the power of signal; $D$ is the module data; $P N$ is the pseudo-random code; $f_{0}$ is the frequency of carrier; $f_{d}$ is the doppler of carrier; $\varphi$ is the phase of carrier; $\hat{f}_{d}$ is the estimation of doppler; $n\left(t_{n}\right)$ is the Gaussian noise in loop, its single side spectral density is $N_{0} ; j\left(t_{n}\right)$ is the broadband Gaussian noise jammer, its single side spectral density is $N_{J}$, and is unrelated with the thermal noise (Gaussian noise)in the receiver, $R_{j n}(k)=0, k \in \mathrm{Z} ; T_{I}$ is the integration time.

After related integration, the outputs of in-phase branch $I(k)$ and quadrature phase branch $Q(k)$ at the $k t h$ loop update time are:

$$
\begin{aligned}
& I(k)=\sqrt{\frac{P}{2}} D(k) R[\Delta \tau(k)] \frac{\sin \left(\pi \Delta f_{d}(k) T_{I}\right)}{\pi \Delta f_{d}(k) T_{I}} \cos (\Delta \theta(k))+N_{I}(k) \\
& Q(k)=\sqrt{\frac{P}{2}} D(k) R[\Delta \tau(k)] \frac{\sin \left(\pi \Delta f_{d}(k) T_{I}\right)}{\pi \Delta f_{d}(k) T_{I}} \sin (\Delta \theta(k))+N_{Q}(k)
\end{aligned}
$$

Where, $\Delta f_{d}(k)$ is the residuals of doppler, $\Delta f_{d}=f_{d}(k)-\hat{f}_{d}(k) ; \Delta \tau(k)$ is the residuals of code phase, $\Delta \tau(k)$ can be set to zero ,when the code loop is tracking; $\Delta \theta(k)$ is the residuals of carrier phase, $\Delta \theta(k)=\Delta \theta(k-1)+2 \pi \Delta f_{d}(k) T_{I}$; when the broadband Gaussian in case; $N_{I}(K)$ and $N_{Q}(k)$ are the superposition of the Gaussian noise, we have:

$$
\begin{gathered}
P_{N_{I}}=P_{N_{Q}}=\frac{N_{0}+N_{J}}{4 T_{I}} \\
R_{N_{I} N_{Q}}(k)=0, \quad k \in \mathrm{Z}
\end{gathered}
$$

To the discriminator, there are a variety of algorithms to choose, cross-over frequency discriminator is the best algorithm for the low $\mathrm{SNR}^{[5]}$, and the method is easily realized with small computation amount. For the slope is proportional to the signal's instantaneous power, the cross-identification algorithm required for normalization, Meanwhile, for the elimination of data bits hopping, the frequency discriminator algorithm is:

$$
e f_{k}=\frac{(\text { cross product }) \times \operatorname{sign}(\text { dot product })}{2 \pi T_{I} P / 2}
$$

Where,

$$
\begin{aligned}
& S_{\text {dot }}=I(k) I(k-1)+Q(k) Q(k-1) \\
& S_{\text {cross }}=Q(k) I(k-1)-I(k) Q(k-1)
\end{aligned}
$$

The function of loop filter is to reduce noise, according to the optimization theory, the optimal loop-filter transfer function is $F_{2}(s)^{[6]}$

$$
F_{2}(s)=\frac{\sqrt{2} w_{n} s+w_{n}^{2}}{K_{d} K_{v} s^{2}}
$$

Where, $K_{d}$ is the gain of discriminator; $K_{v}$ is the gain of NCO; $w_{n}$ is the natural angular frequency of FLL:

Using digital rectangular integral mapping, we can get the transfer function $F_{2}(z)$ of second-order digital FLL in $z$ domain:

$$
F_{2}(z)=\frac{T_{I} w_{n}\left[T_{I} w_{n}+\sqrt{2}\left(1-z^{-1}\right)\right]}{K_{d} K_{V}\left(1-z^{-1}\right)^{2}}
$$




\section{The effect of broadband Gaussian noise jammer to FLL}

According to the mode of FLL, the loop-filter and the discriminator, the effect of broadband Gaussian noise jammer to FLL is studied. The figure 3 shows the mathematical model of second order FLL. Where, $n_{1}(k)$ is the discriminator error casused by themal noise and the jammer, $F_{2}(z)$ is the second order digital filter.

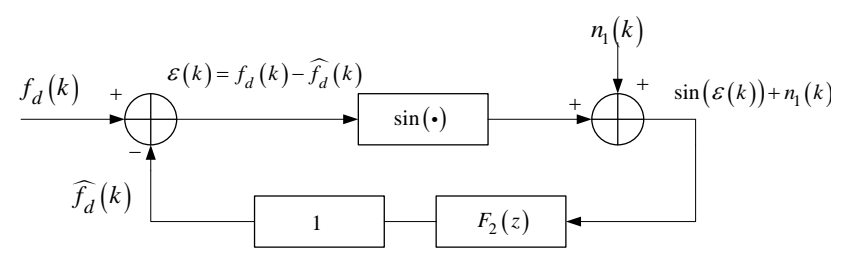

Fig.4 the mathematical model of second order digital FLL

By using a linear approximation, we can get the steady-state error:

$$
\varepsilon(k) \approx f_{d}(k)-F_{2}(z)\left(\varepsilon(k)+n_{1}(k)\right)
$$

According to the colsed-loop transfer function:

$$
H_{F L L}(z)=\frac{F_{2}(z)}{1+F_{2}(z)}
$$

Then the error is:

$$
\varepsilon(k)=\left(1-H_{F L L}(z)\right) f_{d}(k)-H_{F L L}(z) n_{1}(k)
$$

We get the variance of tracking error:

$$
\sigma_{\varepsilon}^{2}=\int_{-T_{I} / 2}^{T_{I} / 2}\left|H_{F L L}\left(e^{j 2 \pi f T_{I}}\right)\right|^{2} S_{n_{1}}(f) d f
$$

Where, $S_{n 1}(f)$ is the spectral density of discriminator's noise ${ }^{n_{1}(k)}$, it is defined as the Eq.(13)

$$
S_{n_{1}}(f)=\sum_{k=-\infty}^{\infty} R_{n_{1} n_{1}}(k) e^{-j 2 \pi f k T_{I}}
$$

Where, $R_{n_{1} n_{1}}(k)$ is the autocorrelation funtion of $n_{1}(k)$ :

$$
R_{n_{1} n_{1}}(k)=E\left[n_{1}\left(k_{0}\right) n_{1}\left(k_{0}-k\right)\right]
$$

When the loop reaches steady state, according to the Eq. (1) Eq. (5), we have:

$$
n_{1}(k) \approx \frac{1}{2 \pi T_{I}}\left\{\begin{array}{l}
\sqrt{\frac{2}{P}}\left[\begin{array}{l}
D(k) N_{Q}(k) \cos [\Delta \theta(k-1)]+D(k-1) N_{I}(k-1) \sin [\Delta \theta(k)] \\
-D(k) N_{I}(k) \sin [\Delta \theta(k-1)]-D(k-1) N_{Q}(k-1) \cos [\Delta \theta(k)]
\end{array}\right] \\
+\frac{2}{P} D(k) D(k-1)\left[N_{I}(k-1) N_{Q}(k)-N_{I}(k) N_{Q}(k-1)\right]
\end{array}\right\}
$$

Then

$$
\begin{gathered}
R_{n 111}(0) \approx\left(\frac{1}{2 \pi T_{I}}\right)^{2}\left[2 \times\left(\frac{2}{P}\right)\left(\frac{\left(N_{0}+N_{J}\right)}{4 T_{I}}\right)+2 \times\left(\frac{4}{P^{2}}\right)\left(\frac{\left(N_{0}+N_{J}\right)}{4 T_{I}}\right)^{2}\right] \\
R_{n 111}( \pm 1) \approx\left(\frac{1}{2 \pi T_{I}}\right)^{2}\left[-\left(\frac{2}{P}\right)\left(\frac{\left(N_{0}+N_{J}\right)}{4 T_{I}}\right)\right] \\
R_{n 1 n 1}(k)=0, \quad \text { else }
\end{gathered}
$$

Substituted $z=e^{j 2 \pi f T_{I}}$ into Eq. (12), we have:

$$
\sigma_{\varepsilon}^{2}=\left(\frac{1}{2 \pi T_{I}}\right)^{2} \frac{1}{2 \pi j} \oint_{|z|=1} H_{F L L}(z) H_{F L L}\left(z^{-1}\right) z^{-1}\left[\begin{array}{l}
\left(\frac{2}{P}\right)\left(\frac{N_{0}+N_{J}}{4 T_{I}}\right)\left(2-z^{-1}-z\right) \\
+2 \times\left(\frac{4}{P^{2}}\right)\left(\frac{N_{0}+N_{J}}{4 T_{I}}\right)^{2}
\end{array}\right] d z
$$

The Eq. (19) can be divided into two parts: 


$$
\begin{gathered}
\sigma_{\varepsilon, 1}^{2}=\left(\frac{1}{2 \pi T_{I}}\right)^{2} \frac{N_{0}+N_{J}}{2 P T_{I}} \frac{1}{2 \pi j} \oint_{|z|=1} H_{F L L}(z) H_{F L L}\left(z^{-1}\right) z^{-1}\left(2-z^{-1}-z\right) d z \\
\sigma_{\varepsilon, 2}^{2}=\left(\frac{1}{2 \pi T_{I}}\right)^{2} \frac{\left(N_{0}+N_{J}\right)^{2}}{2 P^{2} T_{I}^{2}} \frac{1}{2 \pi j} \oint_{|z|=1} H_{F L L}(z) H_{F L L}\left(z^{-1}\right) z^{-1} d z
\end{gathered}
$$

According to the difinition of noise bandwidth:

$$
2 B_{L} T_{I}=\frac{1}{2 \pi j} \oint_{|z|=1} H_{F L L}(z) H_{F L L}\left(z^{-1}\right) z^{-1} d z
$$

Where, $B_{L}$ is the noise bandwidth of FLL.

$$
\sigma_{\varepsilon, 2}^{2}=\left(\frac{1}{2 \pi T_{I}}\right)^{2} \frac{\left(N_{0}+N_{J}\right)^{2}}{P^{2} T_{I}^{2}} B_{L} T_{I}
$$

According to Residue theorem, from Eq.(20) we have:

According to Eq.(8) and Eq.(22), we have:

$$
\sigma_{\varepsilon, 1}^{2} \approx\left(\frac{1}{2 \pi T_{I}}\right)^{2} \frac{N_{0}+N_{J}}{P T_{I}}\left(T_{I} w_{n}\right)^{2}
$$

$$
2 B_{L} T_{I} \approx T_{I} w_{n}\left(\frac{\sqrt{2}}{2}+\frac{1}{2 \sqrt{2}}\right)
$$

Substituting the Eq. (25) into Eq. (24), we have

$$
\sigma_{\varepsilon, 1}^{2} \approx\left(\frac{1}{2 \pi T_{I}}\right)^{2} \frac{N_{0}+N_{J}}{P T_{I}} \times 0.88 \times\left(2 B_{L} T_{I}\right)^{2}
$$

Integrating the Eq.(23) and Eq. (26), we have the thermal noise fibrillation in the presence of broadband Gaussian noise :

$$
\sigma_{t F L L}=\frac{1}{2 \pi T_{I}} \sqrt{\frac{4 B_{L}}{c n r_{e q}}\left(0.88 T_{I} B_{L}+\frac{1}{4 c n r_{e q} \cdot T_{I}}\right)}
$$

Where, the $c n r_{e q}$ is the equivalent carrier-to-noise ratio, $c n r_{e q}=P /\left(N_{0}+N_{J}\right)$.

When there is no jammer, $N_{J}=0$, in the Eq.(27), equivalent carrier-to-noise ratio $c n r_{e q}$ is replace of carrier-to-noise ratio $c n r$. While according to the conclusion of Ref. [5], the thermal noise fibrillation in the presence of broadband Gaussian noise is:

$$
\sigma_{t F L L}=\frac{1}{2 \pi T} \sqrt{\frac{4 F B_{n}}{c n r_{e q}}\left[1+\frac{1}{T c n r_{e q}}\right]}(H z)
$$

Where, $T$ is the integration time; $B_{n}$ is the equivalent noise bandwidth; $c n r_{e q}$ is the equivalent carrier-to-noise ratio; $F=1$, when the CNR is better, $F=2$ when the CNR is poor.

The reason why there is a difference between the Eq.(27) and the Eq. (28) is that the Eq. (28) is derived from the corss-product discriminator, the noise term of the correlation of adjacent update cycle of that is difference to that of this paper (there is a specific analysis in the Ref.[7]).

\section{The Simulation}

Based on the analysis above, the conclusion of this paper is vertified and compared with the conclusion of Ref. [5]. Fig.5 shows the basic principles of simulation. 
Fig. 5 the principles of simulation

The simulation conditions: the dynamic is set to be $1 \mathrm{G}$, the noise bandwidth of FLL is $2 \mathrm{~Hz}$, the equivalent CNR is $31 \sim 51 \mathrm{dBHz}$, the integration time is $2 \mathrm{~ms}$, the discriminator algorithm based on the Eq.(5), the second-order digital filter is taked, the transfer function based on the Eq. (8), where $K_{d}=1, K_{v}=1$. The parameters of the NCO are set properly.

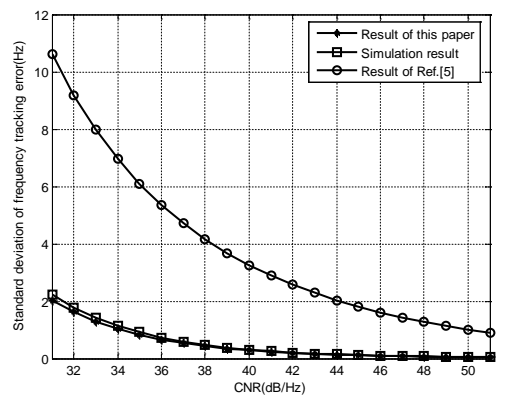

Fig.6 The frequency tracking error according to CNR

Fig. 6 shows the mean of standard deviation of frequency tracking error from 100 times Monte Carlo simulation, the result of this paper and the result from Ref. [5]. It is observed from the figure that the simulation result is consistent with the conclusion from this paper, not consistent with the conclusion from the Ref. [5] by Kaplan.

In addition, the frequency tracking error according to noise bandwidth is vertified. the dynamic is $1 \mathrm{G}$, the CNR is $40 \mathrm{dBHz}$, loop noise bandwidth $B_{L}$ is $2 \mathrm{~Hz} \sim 10 \mathrm{~Hz}$, the others simulation condition is same as that of the first step.

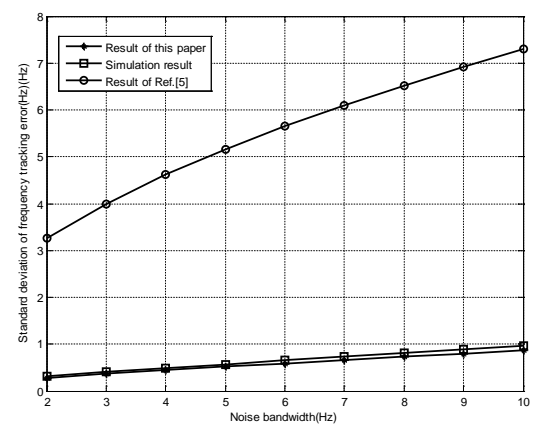

Fig.7 The frequency tracking error according to noise bandwidth

It is observed from figure 7 that the conclusion of this paper is right. Therefore, it is vertified that the analysis about frequency tracking error caused by noise is right. Then, it is got that the conclusion about frequency tracking error caused by noise from Ref. [5] is not poper to be used directly, the specific analysis is needed according to the loop design.

Based on the study above, the noise threshold based on some dynamic of FLL is studied in the broadband Gaussian noise jammer scene. From the Ref. [5], the tracking threshold of FLL is:

$$
3 \sigma_{F L L}=3 \sigma_{t F L L}+f_{e} \leq 0.25 / T
$$


where, $\sigma_{t F L L}$ is thermal noise fibrillation, $f_{e}$ is dynamic stress error , $T$ is integration time.

For the dynamic is $1 \mathrm{G}$, dynamic error is $f_{e}=30 \mathrm{~Hz}$ accordingly. Based on the Eq. (27) , the 1-sigma thermal noise error and threshold are got through simulation, the results are showed in Fig.8.
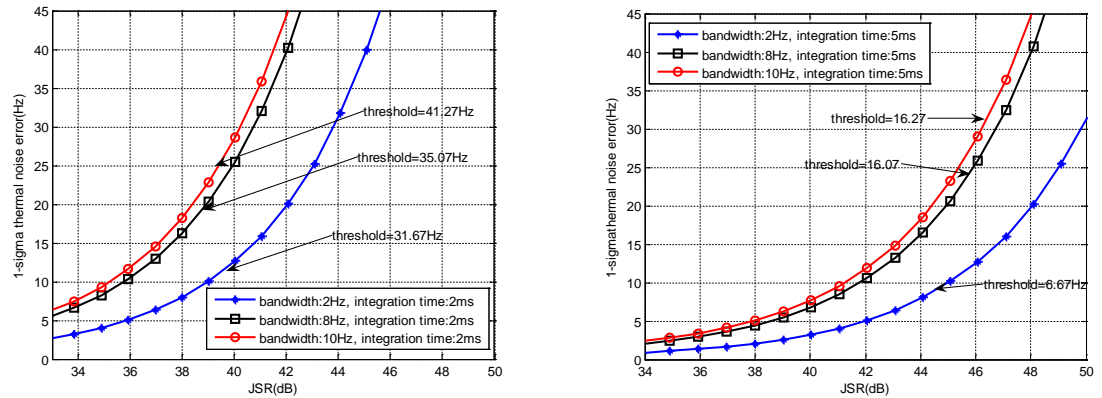

Fig.8 1-sigma thermal noise error and threshold according to JSR

The figure 8 shows that: (1) if the JSR is not greater than 44dB the second order FLL can work properly on the condition that the dynamic is $1 \mathrm{G}$, loop integration time is $2 \mathrm{~ms}$, and the loop bandwidth is $2 \mathrm{~Hz}$; (2) if the JSR is not greater than $41.5 \mathrm{~dB}$ the second order FLL can work properly, with the bandwidth is $8 \mathrm{~Hz}$ and other conditions remain unchanged; (3) if the JSR is not greater than $41.5 \mathrm{~dB}$ the second order FLL can work properly with the bandwidth is $10 \mathrm{~Hz}$ and other conditions remain unchanged.

In addition, we get the JSR threshold with the loop integration time is $5 \mathrm{~ms}$. Based on the study above, we get the table 1 .

Table 1 the JSR threshold with the dynamic is $1 \mathrm{G}$ in the broadband Gaussian noise jammer scene

\begin{tabular}{c|c|c|c}
\hline $\begin{array}{c}\text { Noise } \\
\begin{array}{l}\text { bandwidth(Hz) } \\
\text { Integration time(ms) }\end{array}\end{array}$ & 2 & 8 & 10 \\
\hline 2 & $44(\mathrm{~dB})$ & $41.5(\mathrm{~dB})$ & $41.5(\mathrm{~dB})$ \\
\hline 5 & $44(\mathrm{~dB})$ & $43.8(\mathrm{~dB})$ & $43.5(\mathrm{~dB})$ \\
\hline
\end{tabular}

Based on the simulation result, the following conclusions were obtained:

(1) For the combined effect of the dynamic stress error and noise error, there is no significant improvement for the loop anti-jamming performanc by increase the integration time. The dynamic and anti-jammer performances of receiver loop are better when the integration time is $2 \mathrm{~ms}$ to those when the integration time is $5 \mathrm{~ms}$.

(2) If the integration time keeps unchanged, increaseing the loop noise bandwidth makes the anti-jamming performance deteriorates. The main reason is that the power of jammer into FLL will increase with the loop noise bandwidth increasing.

Those conclusions above are based on the assumption that the dynamic is 1G. The noise bandwidth, integration time should be set according to the signal features and environmental requirements.

\section{Conclusion}

Based on considering the key parts of frequency-locked loop, the effect of broadband Gaussian noise on the tracking error of frequency-locked loop is studied in depth, and the colsed form solution is derived. Then, the conclusion that the thermal noise fibrillation of FLL should be analysised according to the specific loop design is got. Based on the study above, the noise threshold of FLL based on some dynamic is studied in the broadband Gaussian noise jammer scene, and some conclusions are got. The results of this paper have certain reference for the development of anti-jamming navigation receiver. 


\section{References}

[1] JIANG Yi, ZHANG Shufang, HU Qing, et al. A Low Complexity Design of GPS Carrier Tracking Loop [J]. ACTA ELECTRONICA SINICA, vol.38, No.12, pp: 2822-2826, 2010.

[2] WANG Jun, LI Jiaqi, WU Siliang. Tracking Error Analysis of FLL-Assisted-PLL [J]. Transactions of Beijing Institute of Technology, vol.31, No.7, pp: 838-843, 2011.

[3] Zhuang W. Performance analysis of GPS carrier phase observable [J]. IEEE Transactions on Aerospace and Electronic Systems, vol.32, No.2, pp: 754-767, 1996.

[4] Tang Junjie, Xue Lei, Li Jianqiang. Analysis and Simulation of the Effects of Broadband Noise Interference on GPS M Code Receiver [J]. Ship Electronic Engineering, vol.30, No.8, pp: 74-77, 2010.

[5] Kaplan E D. Understang GPS Principles and Applications [Z]. Beijing: publishing house of electronics industry, 2006:164-166.

[6] Jwo D J. Optimisation and sensitivity analysis of GPS receiver tracking loops in dynamic environments [J]. IEE Proceedings-Radar, Sonar and Navigation. 2001, 148(4): 241-250.

[7] WANG Jun. Reaseach on Key Technologies of the Satellite Navigation Positioning Receiver Signal Processing [D] .Beijing: Beijing Institute of Technology, 2011. 